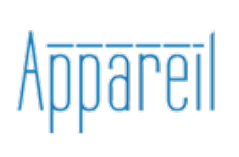

Appareil

$15 \mid 2015$

L'art dans le tout numérique

\title{
Walter Benjamin : la perspective comme fantasmagorie
}

Jean-Louis Déotte

\section{OpenEdition}

1 Journals

Édition électronique

URL : http://journals.openedition.org/appareil/1971

DOI : 10.4000/appareil. 1971

ISSN : 2101-0714

Éditeur

MSH Paris Nord

Référence électronique

Jean-Louis Déotte, «Walter Benjamin : la perspective comme fantasmagorie », Appareil [En ligne], 15 | 2015, mis en ligne le 06 février 2014, consulté le 01 août 2020. URL : http://journals.openedition.org/ appareil/1971; DOI : https://doi.org/10.4000/appareil.1971

Ce document a été généré automatiquement le 1 août 2020.

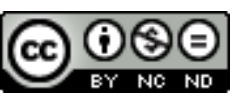

Appareil est mis à disposition selon les termes de la Licence Creative Commons Attribution - Pas d'Utilisation Commerciale - Pas de Modification 4.0 International. 


\title{
Walter Benjamin : la perspective comme fantasmagorie
}

\author{
Jean-Louis Déotte
}

1 Je ne pouvais pas désirer salle ${ }^{1}$ plus adaptée à mon discours sur Benjamin et la perspective comme fantasmagorie que cette dépendance d'un passage urbain parisien. Si la galerie Colbert a plus d'élégance perspectiviste, la galerie Vivienne l'a toujours emporté sur sa sœur rivale, pour des raisons qui tiennent à l'exposition réussie des marchandises de luxe. En deux temps, le xix e siècle parisien a vu surgir deux architectures que tout oppose : en son premier tiers, les passages, en un second, les perspectives haussmanniennes. Si les passages sont analysés par W. Benjamin comme l'élément le plus éloquent de la série "maison du rêve collectif ", caractérisant au mieux le siècle, à la suite des gares, des établissements thermaux, des égouts, etc., les perspectives haussmanniennes, au contraire, appartiennent à un passé qui ne cesse de revenir, à l'éternel retour du Même. Ce sont des réalités quasi spectrales même si, au contraire des passages, elles sont à ciel ouvert. On peut se demander alors si les perspectives urbaines ne sont pas toujours des compléments oniriques des passages qui se sont mondialisés depuis Paris².

2 Pourtant, sur le plan de ce qu'on pourrait appeler une cosmétique générale, si l'on développe une analyse en termes d'appareillage de l'apparaître, perspective et passage urbain sont bien des appareils. En termes phylogénétiques, la perspective inaugura une famille prodigieuse d'appareils projectifs dont le dernier en date, le cinéma, synthétise tous les autres : «Le cinéma : démêlement (résultat?) de toutes les formes de vision, de tous les rythmes et de tous les temps préformés dans les machines actuelles, de telle sorte que tous les problèmes de l'art actuel ne peuvent trouver leur formulation définitive qu'en corrélation avec le film $»^{3}$.

3 Les appareils sont indissociables de leurs effets dans le réel, ceux de la perspective à point de fuite sont bien connus, surtout quand ils reviennent sous la forme urbaine imposée par Haussmann. S'il est vain de dissocier peinture, sculpture, architecture, voire théâtre dans le cas de la perspective, les effets de réel de l'appareil spécial qu'est le passage urbain furent plus immédiatement économiques et psycho-sociaux, puis 
tardivement artistiques (le surréalisme). Mais ces premiers aspects tardent encore à être systématiquement explorés pour la perspective, je veux parler ici de la question ontologique du partage sujet/objet et de celle, politique, de la République.

4 Mais puisque aujourd'hui, s'agissant de la perspective, depuis les travaux fondateurs de Panofsky et leur refondation phénoménologique par Damisch, en les croisant avec la grande étude de Lefort sur la politique républicaine de Machiavel, on peut mettre en rapport l'invention du géométral perspectif et la refondation de l'espace public, à la suite de W. Benjamin, il devient possible d'effectuer la même opération s'agissant d'un appareil qui n'est plus projectif, mais immersif et inclusif (le passage urbain), en se demandant quels furent ses effets sur la singularité quelconque et sur l'être-ensemble politique. Si l'appareil perspectif a permis la représentation d'un espace où potentiellement tous les points de vue se valaient, au sens où connaissant le point de vue principal, on peut imaginer tous les autres, le surgissement de ce site, qu'on oppose à la hiérarchie des lieux aristotéliciens, implique sur le plan social-politique l'affirmation du principe d'égalité. Que reste-t-il de ce principe d'égalité quand l'espace devient immersif du fait du succès des passages urbains? Qu'en est-il du sujet au sens philosophique du terme?

5 Si Benjamin n'a pas étudié l'appareil perspectif, il se rattrape avec la photographie et le cinéma, sa philosophie étant d'ailleurs davantage d'essence photographique ${ }^{4}$. On peut ajouter que W. Benjamin a littéralement manqué la première Renaissance (l'humanisme véritable) pour des raisons qui sont au premier abord contingentes (sa prédilection pour Stendhal et les Chroniques italiennes). Mais pas seulement.

6 En effet, dès Le programme de la philosophie qui vient ${ }^{5}$ qui peut être considéré comme un texte de jeunesse, dans lequel W. Benjamin critique la notion kantienne d'expérience à partir de sa lecture des néo-kantiens, il est évident que le cadre de la Critique de la raison pure lui apparaît comme beaucoup trop étroit pour rendre compte du jugement esthétique et de la théologie. À cette époque, W. Benjamin ne doit pas avoir lu la Critique du jugement: la conception de l'expérience qu'il attribue à Kant est intrinsèquement confondue avec celle de la science, elle est objectivisante. S'il s'en prend à l'opposition sujet/objet, c'est qu'il réduit le sujet au sujet transcendantal de la connaissance et l'objet à la chose quantifiée et mesurée.

7 Mais c'est dire que si ce kantisme est la philosophie qui problématise philosophiquement les conditions de la connaissance scientifique sur une base inévitablement perspectiviste, le marginaliser comme le fait $\mathrm{W}$. Benjamin conduit inévitablement à une relativisation de la perspective. D'ailleurs l'opposition sujet/objet sera proprement déconstruite, c'est ce qu'annonce le Programme: la philosophie qui vient consistera en une déconstruction toujours plus approfondie de l'opposition, au profit de ce qui est au milieu, au profit de ce qui est entre sujet et objet et les rend possibles.

8 Bref, très tôt, dès ses analyses d'esthétique (Fragments), la recherche benjaminienne portera sur ce qui relève du ni, ni: ni sujet, ni objet, donc sur ce qui précède la différenciation en objet et sujet. Ce qui est ainsi au milieu portera au fil des œuvres différents noms. Dans les Fragments d'esthétique, c'est l'imagination qui joue ce rôle. On sait le mal qu'avait eu Kant, entre la première et la seconde version de la première Critique pour définir l'imagination comme faculté de connaissance, entre les formes de la sensibilité pure et les concepts de l'entendement. Tout se passe au contraire chez W. Benjamin comme s'il fallait partir d'elle, avant toute différenciation entre les autres 
facultés de la connaissance qui, à des degrés divers, mettent en forme la matière du sensible. Ne perçoit-on pas, ne connaît-on pas en la mettant en forme, d'une manière ou d'une autre? Ce qui a pour conséquence que l'imagination n'est plus une faculté formatrice, arrimée de près ou de loin à l'appareil perspectif, mais une puissance que tous les appareils projectifs et inclusifs supposeraient déjà avant d'être des déterminations de la « surface absolue » ou de la fantasmagorie originaire.

L'imagination n'est plus la Einbildungskraft kantienne, cette puissance de mise en forme, mais une puissance de déformation, de passage, bien que non-destructrice, ce qui suppose des entités déjà-là sur lesquelles elle s'applique, une sorte de patrimoine dont tout enfant hériterait du simple fait de naître dans une culture donnée à une époque donnée. La production artistique consisterait alors en une sorte de dissolution des formes acquises, par exemple la culture des "vainqueurs de l'histoire», laquelle est inévitablement la nôtre...

Si l'imagination artistique ne met plus en forme des matériaux artistiques, c'est que le rapport matière/forme ne peut plus être entendu à la manière platonicienne ou aristotélicienne, car entre la matière et la forme, entre le contenu et le contenant, il y a plutôt chez W. Benjamin une continuité, un passage, un rapport d'expression, d'évocation, de rappel au présent (Vergegenwärtigung). La dimension majeure de l'imagination serait plutôt le passé. On pourrait conclure, classiquement, que la mémoire étant la mère des Muses, il n'est pas extraordinaire que la production artistique soit greffée sur elle. Mais chez W. Benjamin, il y a des époques de la mémoire, qu'il faudra distinguer. Comme il y aura des époques de la transmission. La matière artistique serait alors de l'ordre de la remémoration.

11 À l'opposé, l'appareil perspectif en s'imposant aux arts à partir du xve siècle, en faisant ainsi époque, privilégia l'un d'entre eux : le dessin. Le dessin, élargissant sans limites ses frontières, en devenant le disegno des Traités, fut alors à la Renaissance le véritable nom de la Forme, de l'Idée entendue comme projet, mais aussi comme archive, mémoire, idéalité, trace, etc. Bref, il se subordonna la matière et en particulier la couleur, laquelle devint une affaire de teinturier. C'est-à-dire de l'art des teinturiers (au sens que prit ce mot à Florence où s'imposa l'opposition sociale-politique entre arts majeurs et arts mineurs).

Pour reprendre le terme qu'utilise Simondon pour caractériser le rapport matière/ forme chez Aristote : soit la notion d'hylémorphisme, il y eut bien dans la conception de la Forme que développèrent les auteurs renaissants des Traités, un hylémorphisme spécifique, s'agissant déjà de la couleur, mais peut-être plus essentiellement de ce qui était digne d'être représenté par rapport à une sorte d'irreprésentable social et politique, soumis l'un et l'autre au formatage de l'appareil perspectif, nécessairement premier et essentiel, comme le montre Alberti dans Della Pittura, même s'il lui donne comme finalité la narration ${ }^{6}$.

Or si l'hylémorphisme aristotélicien (dont dépend une bonne partie de la métaphysique) est devenu un schème universel de pensées, il a, de ce fait, justifié et mondialisé un rapport social qui lui n'avait rien d'universel : le rapport maître/esclave, car dans le cadre antique, le maître était bien la forme qui soumettait en la finalisant la matière-esclave. Dès lors, s'il y eut quelque chose comme un hylémorphisme projectif renaissant, que tente de problématiser Panofsky dans Idea ${ }^{7}$, cet hylémorphisme devait bien justifier et mondialiser un autre rapport social et politique de domination, celui que la révolte prolétarienne des Ciompi au $\mathrm{XIV}^{\mathrm{e}}$ siècle tenta d'abattre ${ }^{8}$. Si le principe 
d'égalité promu par l'appareil perspectif était bien le sol de toute l'histoire occidentale à venir, la contradiction éclata à Florence en 1378. Alessandro Stella écrit : « La requête d'une reconnaissance légale d'un art propre, en effet, bouleversait l'ordre social, économique et même politique. Il était moins question de sanctionner juridiquement l'apparition d'une nouvelle et énième corporation que de reconnaître aux "soumis" (les Ciompi), aux sans-droits, aux sans-paroles, aux sans-plumes, l'autonomie, la dignité d'un travail considéré comme un art à part entière, la possibilité d'avoir voix au chapitre dans les affaires de la cité. ${ }^{9}$

Chez W. Benjamin, la question du sujet est implicitement celle de la perspective, dont le sort est indissociable de celui du roman, lequel naît selon lui, avec Cervantès et s'achève avec l'écriture de montage d'Alexanderplatz de Döblin ou du cinéma. Si les perspectives urbaines parisiennes furent le dada d'Haussmann et de Louis-Napoléon (des peluches pour l'œil, du kitsch ${ }^{10}$ ), c'est du fait d'un certain fétichisme, d'un narcissisme secondaire confinant à l'autoérotisme du roman qui est toujours «le mémorial d'un moi " selon Benjamin. Le supposé sujet de la perspective a été substantifié par la psychologie sous la forme du Moi, lequel cultive son intériorité comme tout individu "particulier» au temps de Louis-Philippe cultivant ses chers " souvenirs » dans un appartement transformé en étui.

Perspective et appartement-étui deviennent pour Benjamin des fantasmagories secondaires, complémentaires, spatiales, des rêveries collectives et individuelles. Leur correspondrait sur le plan de la temporalité : le culte de la Nouveauté (l'ultramodernité de la Mode) qui liquide l'évocation du passé, mais aussi l'enfermement dans l'éternel retour du Même, soit le retour incessant des archaïsmes. Les nouvelles apportées par les journaux ne sont plus assimilables, comme le sont les événements du passé pour la narration plébéienne, car l'information journalistique relève de l'objectivation du monde. Dès lors, ces fantasmagories secondaires déterminent la sensibilité commune prégnante au $\mathrm{XIX}^{\mathrm{e}}$ siècle. Ce sont des formes d'aliénation, déterminantes pour W. Benjamin dont le projet d'écriture (Paris, capitale du XIXe siècle) est essentiellement politique : comprendre comment les masses urbaines du siècle passé auront pu être mobilisées par les partis totalitaires $\mathrm{du} \mathrm{xx}^{\mathrm{e}}$ siècle, puisque chaque "époque rêve la suivante ». Quelle est la détermination psycho-sociale de la foule qui parcourt les passages? La figure du «sujet» politique a-t-elle un avenir entre objectivation du monde et fantasmagorie secondaire?

Si le projet de W. Benjamin n'avait concerné, comme l'affirme M. Berdet ${ }^{11}$, que la description du capitalisme "le plus avancé " au XIX siècle, il n'aurait pas pris comme objet d'étude Paris, mais Londres, l'avant-garde du capitalisme comme le rappelle Engels ${ }^{12}$. La question politique devient alors celle-ci : qu'est-ce qui a pour W. Benjamin fondamentalement distingué Paris de Londres sur le plan architectural et urbanistique? Accessoirement: le percement autoritaire des perspectives haussmanniennes ${ }^{13}$. Essentiellement: les passages urbains ${ }^{14}$. Parce que ce sont des formes-matrices de l'immersion et donc du rêve collectif, c'est-à-dire de la fantasmagorie. Des générateurs spécifiques de fantasmagories secondaires prétotalitaires. On sait que Giedion qui avait permis à W. Benjamin de découvrir la technicité de l'architecture pré-moderne française ${ }^{15}$ ne dit rien des passages qui en relèvent pourtant, du fait de la caractéristique de leurs matériaux (la fonte, le verre), mais fait l'éloge de l'urbanisme londonien pour avoir su transformer les jardins des grandes propriétés de l'aristocratie en squares publics ${ }^{16}$. 
17 Il y aurait donc selon W. Benjamin un hylémorphisme caractéristique de la prémodernité où les passages urbains comme formes déterminantes s'imposeraient à un matériau psycho-social, la masse des chalands d'un côté, le particulier enkysté dans son appartement de l'autre. Comme selon lui, il y a une continuité expressive entre la forme et le contenu, un passage permanent, la forme a la substance du rêve et le contenu celle des processus physiologiques, des humeurs. Comme il a été dit plus haut, il n'y a plus prévalence chez W. Benjamin de la forme sur le contenu, mais réversibilité : le monde du passage est une construction technique «extérieure» qui informe la masse enfermée dans une rêverie collective, laquelle, en retour, annexe ce qui lui était pourtant extérieur. L'architecture industrielle des passages est alors comme absorbée par la masse. Entre la forme et le contenu, il y a bien une inclusion réciproque, un passage continuel. Le passage urbain réalise l'essence de la faculté humaine essentielle : l'imagination, laquelle consiste en un passage permanent. On peut affirmer par conséquent que W. Benjamin a subverti le matérialisme dialectique de Marx, puisqu'il ne pense plus en termes d'infrastructure déterminant une superstructure socioculturelle (c'est d'ailleurs son défi à Giedion ${ }^{17}$ ), ce qu'annonçait déjà son étude sur le collectionneur et historien Fuchs ${ }^{18}$. Et en termes contemporains, cela reviendrait à dire que certes, c'est le medium qui détermine le message, parce que l'inverse est tout aussi vrai. Les perspectives haussmanniennes subissent le même sort : ce sont des passages à ciel ouvert, ce qui est vrai pour la ville dans son ensemble. On peut considérer que dorénavant l'humanité vit au fond d'un océan vertical, ce qu'illustre le transformisme radical de Grandville. Ou l'atmosphère aquatique du Passage de l'Opéra selon Aragon ${ }^{19}$.

La philosophie de la surface qu'on doit considérer comme un milieu débouche sur un immanentisme radical, dont on trouvera un écho dans L'économie libidinale de Lyotard, chez lequel ne subsiste qu'une surface (la peau) parcourue par des affects d'intensité différente. Que devient alors la perspective pour laquelle il y a nécessairement une transcendance : la Nature ? La Nature qui est dans le collimateur de l'appareil, la Nature qu'il faut perdre pour l'objectiver?

19 La masse parcourant les passages devient fétichiste, entre rêveries et processus physiologiques (les humeurs). Ce n'est pas que le fétichisme de la marchandise cher à Marx s'impose à elle, mais plutôt que la fantasmagorie originaire impose une nouvelle forme de spectacularisation du fait de l'invagination des rues. C'est comme si, dans un processus d'involution, la masse, qui ne se partage plus en classes sociales, s'enroulait dans son propre rêve et devenait l'esclave de ses humeurs, se recroquevillait dans son propre corps collectif. Il est essentiel de rappeler que Benjamin n'utilise pratiquement pas le terme marxien d'idéologie, mais celui de fantasmagorie ou de rêverie collective, qui sont des déterminations psycho-sociales de l'imagination. La notion d'idéologie est indissociable d'un autre appareil projectif, la camera obscura, comme le rappelait S. Kofman dans Camera obscura. De l'idéologie, Galilée, 1973.. La camera obscura suppose encore une transcendance, celle de la source de lumière, qui est bien réelle. Ce qui n'est évidemment pas le cas de la fantasmagorie originaire, qu'il faut considérer comme la substance de la sensibilité collective. Tout se passe pour Benjamin comme si originairement l'espace du corps du collectif était un espace d'image, avait la consistance de l'image. Il y eut certes au XIX ${ }^{e}$ siècle une pratique d'engins optiques générant des images spectrales à la suite du succès considérable du dispositif de Robertson comme le rappelle Max Milner ${ }^{20}$. Mais on ne peut pas réduire la fantasmagorie originaire à ces productions spectrales qui n'eurent qu'un temps. Quand 
Benjamin écrit que le $\mathrm{XIX}^{\mathrm{e}}$ siècle a inventé la préhistoire (Urgeschichte), il ne s'agit pas trivialement de la discipline scientifique, mais d'affirmer que le xix ${ }^{e}$ siècle s'est développé au plus près de l'originaire. Son anthropologie est une ontologie et une méthodologie, qui ne doivent rien à la théorie des archétypes de Jung. Sa thèse serait la suivante : en innervant la sensibilité collective par des appareils esthétiques comme le cinéma, nécessairement techniques, le collectif moderne n'a jamais été aussi près de sa vérité structurelle, c'est-à-dire de son émancipation. C'est alors que les vérités de la science la plus contemporaine confirment les intuitions des talmudistes.

La notion de fantasmagorie originaire est évidemment proche du fantasme originaire de la psychanalyse, car comme lui, ce n'est pas une réalité historique, mais transhistorique. On a trop longtemps confondu fantasme originaire et fantasme des origines selon Laplanche et Pontalis ${ }^{21}$ pour ne pas être prudent: Benjamin consacre assez de temps à critiquer Jung pour nous rassurer. La fantasmagorie originaire est seulement structurante, ce ne sont pas les prétendus mythes fondateurs de l'humanité. Elle procède par invariants, lesquels se manifestent historiquement et empiriquement. Le modèle de la boîte et du désemboîtement continu sont des marques de fabrique de la pensée benjaminienne. Celui de la compénétration des espaces prolonge cette logique. De là sa caractérisation des villes (Paris, Moscou, Naples) selon que la compénétration des espaces privés et publics est assumée ou non. Quand elle est parfaitement réussie comme à Naples, car on trouve dans les rues de Naples des scènes d'une intimité qui serait invraisemblable partout ailleurs, c'est semble-t-il du fait de l'élection d'un matériau constructif spécial à Naples, la pierre de lave, dont la porosité est la clef de l'échange des réalités les plus opposées. Il y a alors parfaite adéquation entre la métamorphose de la forme (ses différents états) et la réalité du matériau. Il y a des matériaux qui permettent la réversibilité des échanges entre états différents de la forme et d'autre pas. On sait que la réflexion de Benjamin porte sur la question des seuils, or toutes les sociétés humaines ont accordé une importance spéciale aux seuils, c'est la question anthropologique des rites de passage (Van Gennep) qui nous introduit à la temporalité et à son traitement social. Benjamin peut écrire à la fois que notre «époque est pauvre en expériences du seuil» et que la modernité architecturale, en construisant des maisons de verre, a accompli une véritable révolution. Il peut alors sembler paradoxal de rapprocher la pierre de lave et la paroi de verre, mais l'une et l'autre sont des déterminations de la membrane de transformations dans une topologie. Le seuil est donc le nom benjaminien de la membrane topologique. Pour Benjamin, la transparence («révolutionnaire») du verre est du même ordre que la porosité de la pierre de lave. Il y a donc bien des «échangeurs» topologiques qui permettent de structurer la fantasmagorie originaire, laquelle échappe ainsi au primat freudien de la libido. D'autant qu'un fantasme chez Freud, c'est toujours un scénario érotique où la place du « sujet » reste indéterminée. Benjamin n'était pas un grand freudien!

Il y aurait donc une anthropologie benjaminienne à problématiser, où la question de la technique comme innervation du corps collectif, la question du double qui n'est pas nécessairement un spectre, le « communisme primitif» (le village Hottentot du texte sur Naples), etc., seraient des pièces essentielles. 


\section{BIBLIOGRAPHIE}

Aragon Louis, Le paysan de Paris, Paris, éditions de la Nouvelle Revue française, 1926.

Barot E., 1378 ou l'émergence de la question moderne du sujet révolutionnaire, tome I : « La révolte des Ciompi », Toulouse, Les Réveilleurs de la nuit, 2013.

Benjamin Walter, CEuvres complètes, tome I, Folio, 2000-2011.

Benjamin Walter, Paris, capitale du XIX ${ }^{\mathrm{e}}$ siècle, Jean Lacoste (trad.), Paris, éditions du Cerf, 1989.

Berdet Marc, Fantasmagories du capital : L'invention de la ville-marchandise, Paris, Zones, 2013.

Chadych Danielle, Leborgne Dominique, L'Atlas de Paris, évolution d'un paysage urbain, Parigramme, 2007.

Déotte Jean-Louis, Walter Benjamin et la forme plastique, Paris, L'Harmattan, 2012.

Déotte Jean-Louis, L'Homme de verre : esthétiques benjaminiennes, Paris/Montréal, L'Harmattan, 1997.

Engels Friedrich, La situation de la classe laborieuse en Angleterre, Éditions sociales, 1845.

Geist Johann Friedrich, Le Passage. Un type architectural au XIX ${ }^{e}$ siècle, Mardaga, 1995.

Giedion Siegfried, Espace, temps, architecture [1940], Paris, Denoël Gonthier, 1978.

Giedion Siegfried, Construire en France, construire en fer, construire en béton [1928], Paris, éditions de la Villette, 2000.

Laplanche, Pontalis, Fantasme originaire, fantasme des origines, origine du fantasme, Revue française de psychanalyse, 1964.

Machiavel Nicolas, Histoires florentines, livre III, Pléiade, 1957.

Milner Max, La fantasmagorie, Paris, Presses universitaires de France, collection « Écriture », 1982.

Panofsky, Idea [1924], traduit de l'allemand par Henri Joly (trad.), préface de Jean Molino, éditions Gallimard, 1989.

\section{NOTES}

1. Une salle de l'Institut national de l'Histoire de l'Art, passage Colbert, Paris. Colloque sur la perspective, décembre 2013.

2. Geist Johann Friedrich, Le Passage. Un type architectural au XIX ${ }^{e}$ siècle, Mardaga 1995.

3. Benjamin Walter, Paris, capitale du $\mathrm{XIX}^{\mathrm{e}}$ siècle, Jean Lacoste (trad.), Paris, éditions du Cerf, 1989 , p. 412.

4. C'est son ami Kracauer qui tirera les conséquences de ses intuitions sur le cinéma (Théorie du film).

5. Benjamin Walter, Euvres complètes, tome I, Folio, 2000-2011.

6. Le traitement des formes colorées, l'absence de délinéation des figures de la Chapelle du Carmine par Masaccio, si important pour Lyotard (Discours, Figure), auraient donc été des exceptions.

7. Panofsky, Idea [1924], traduit de l'allemand par Henri Joly (trad.), préface de Jean Molino, éditions Gallimard, 1989. 
8. Machiavel Nicolas, Histoires florentines, livre III, chapitres 1 à 21, tome 2, Pléiade, 1957.

9. Alessandro Stella, La Révolte des Ciompi. Les hommes, les lieux, le travail, éditions de l'EHESS, 1993. Cité par E. Barot, 1378 ou l'émergence de la question moderne du sujet révolutionnaire. I : La révolte des Ciompi, Toulouse, Les Réveilleurs de la nuit, 2013.

10. «Le goût d'Haussmann pour les perspectives représente une tentative pour imposer des formes d'art à la technique (urbanistique). Cela conduit toujours au kitsch.", Walter Benjamin, Paris, Capitale du XIX ${ }^{e}$ siècle, Paris, Le Cerf, p. 1.

11. Berdet Marc, Fantasmagories du capital : L'invention de la ville-marchandise, Paris, Zones, 2013.

12. Engels Friedrich, La situation de la classe laborieuse en Angleterre, Editions sociales, 1845.

13. Comme le montrent les auteurs de L'Atlas de Paris, Évolution d'un paysage urbain (D. Chadych, D. Leborgne), on a beaucoup exagéré le «perspectivisme » d'Haussmann au détriment de ce qui était impératif pour lui : l'alignement des façades et la multiplication des repères visuels. Ainsi la fontaine Saint-Michel communique-t-elle avec la Sainte-Chapelle alors que le boulevard SaintMichel n'est pas dans l'axe du boulevard Sébastopol, etc.

14. Il faut noter qu'il y eut peu de passages construits à Londres même, une vingtaine en comptant ceux qui restèrent à l'état de projets. Mais chaque grande cité industrielle britannique eut les siens (cf. Geist, 1995).

15. Giedion Siegfried, Construire en France, construire en fer, construire en béton [1928], Paris, Éditions de la Villette, 2000.

16. Giedion Siegfried, Espace, temps, architecture [1940], Paris, Denoël Gonthier, 1978.

17. Lire à ce sujet notre Walter Benjamin et la forme plastique, Paris, L'Harmattan, 2012.

18. Notre L'Homme de verre : esthétiques benjaminiennes, Paris/Montréal, L'Harmattan, 1997.

19. Aragon Louis, Le paysan de Paris, Paris, édition de la Nouvelle Revue française, 1926.

20. Milner Max, La fantasmagorie, Paris, Presses universitaires de France, collection «Écriture ", 1982.

21. Laplanche, Pontalis, Fantasme originaire, fantasme des origines, origine du fantasme, Revue française de psychanalyse, 1964.

\section{RÉSUMÉS}

L'appareil perspectif joue un rôle secondaire chez Benjamin, mais c'est qu'il dépend d'un autre appareil, urbain celui-là, apparu au XIX siècle à Paris : le passage. Le paradoxe, et l'anachronisme, sont donc les suivants : les perspectives haussmanniennes sont intelligibles à partir de ces rues invaginées qui eurent un grand succès sous Louis-Philippe! Même si tout oppose ces deux réalités urbaines, elles ont en commun d'être des lieux de passage qui, de ce fait, permirent à la faculté humaine qui est essentielle pour Benjamin de s'épanouir : l'imagination. L'imagination qui est pour lui la «faculté » par excellence du passage. C'est-à-dire de la déformation, toujours réversible, entre forme et matière, contenant et contenu. 
INDEX

Mots-clés : Walter Benjamin, passage urbain, perspective, Haussmann, imagination, fantasmagorie collective

\section{AUTEUR}

\section{JEAN-LOUIS DÉOTTE}

Philosophe français, professeur à l'université Paris 8 